\section{Damien Contou Nicolas de Prost}

\title{
Golden sputum
}

Received: 16 April 2015

Accepted: 17 April 2015

Published online: 14 May 2015

(C) Springer-Verlag Berlin Heidelberg and ESICM 2015

\section{Contou $(\bowtie) \cdot$ N. de Prost}

Service de Réanimation Médicale, CARMAS research group, Groupe Henri Mondor-Albert Chenevier, Assistance PubliqueHôpitaux de Paris, UPEC-Université Paris-Est Créteil Val de Marne, Créteil, France e-mail: damien.contou@hmn.aphp.fr Tel.: +33-1-49-81-23-91

A 26-year-old man with known homozygous sickle cell disease was admitted to the intensive care unit for a febrile acute respiratory failure related to acute chest syndrome. Upon admission, he had profuse yellowish sputum (Fig. 1) and pulmonary auscultation revealed bilateral basal crackles. Chest X-ray and CT scan depicted typical bilateral alveolar consolidations (Fig. 2). Blood and sputum cultures were sterile and urinary antigen tests were negative for both Streptococcus pneumoniae and Legionella pneumophila. The evolution was favorable after red blood cell exchange transfusions and antibiotic therapy including cefotaxime and spiramycin. Acute chest syndrome is the leading cause of intensive care admission in patients with sickle cell disease and is defined as a new infiltrate on chest X-ray in conjunction with one other new symptom or sign among chest pain, cough, wheezing, tachypnea, and/or fever. Golden sputum is a classical and pathognomonic sign of acute chest syndrome.

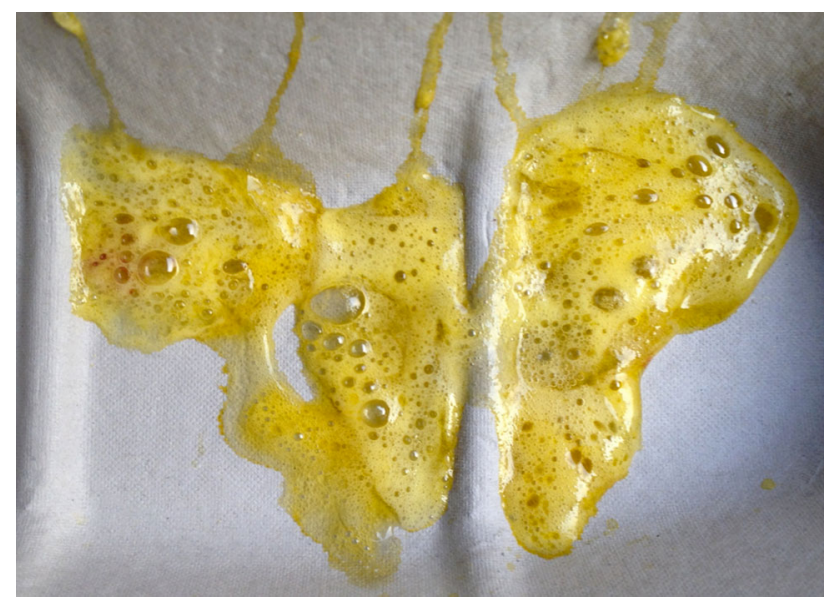

Fig. 1 Golden sputum

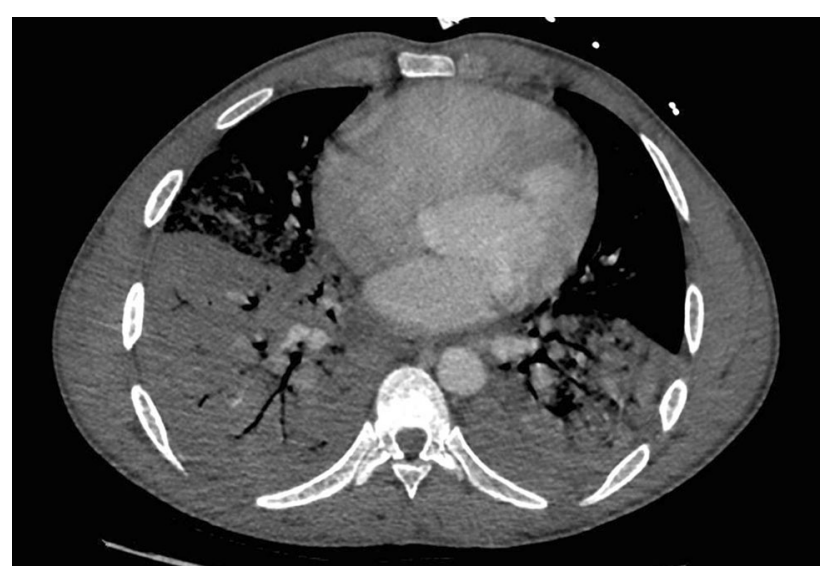

Fig. 2 Chest CT scan depicting bilateral alveolar consolidations typical for the acute chest syndrome in sickle cell disease

Conflicts of interest The authors have no conflict of interest and no financial disclosure to declare. 